\title{
Comparison of Biochemical Recurrence-Free Survival Between Periprostatic and Pelvic Lymph Node Metastases of Prostate Cancer
}

International Journal of Surgical Pathology 2I (4) 352-357

(c) The Author(s) 2013

Reprints and permissions:

sagepub.com/journalsPermissions.nav

DOI: $10.1177 / 10668969 \mid 3482729$

ijs.sagepub.com

(SAGE

\author{
Nilda González-Roibón, MD', Jeong S. Han, MD', Stephen Lee, MD', \\ Zhaoyong Feng, MS', Sehbal Arslankoz, MD², Nathaniel Smith, MD', \\ Philip M. Pierorazio, MD', Elizabeth Humphreys, MS', Theodore L. Deweese, MD', \\ Alan W. Partin, MD, PhD', Trinity J. Bivalacqua, MD, PhD', Misop Han, MD', \\ Bruce Trock, PhD', and Georges J. Netto, MD'
}

\begin{abstract}
Objective. To assess the pathologic characteristics and prognostic significance of periprostatic lymph node (LN) metastasis of prostate cancer. The latter was performed by comparing biochemical recurrence (BCR)-free survival in cases of periprostatic LN metastasis versus matched patients showing pelvic LN metastasis. Methods and Materials. We identified I 5 patients who underwent radical prostatectomy in our institution (1984-20II) showing positive periprostatic and negative pelvic LN with available follow-up information (group I). These patients were matched I:2 to patients with positive pelvic LN (group 2) for pertinent clinicopathologic parameters. Results. Main locations of positive periprostatic LN were posterior base and mid posterolateral. Overall higher rate of positive margins, smaller LN, and metastasis size were encountered in group I compared with group 2.At 5 years postprostatectomy, $69 \%$ of patients in group I were free of BCR, whereas $26 \%$ of those in group 2 remained BCR free, suggesting that patients with periprostatic node metastasis appeared to have a lower risk of BCR. However, the difference was not statistically significant $(P=.072)$. The same was true when adjusted for the effect of prostate-specific antigen, surgical margin status, size of LNs, size of metastasis, age, and year of surgery. Conclusion. Patients with periprostatic node metastasis may have a lower risk of BCR compared with those with metastasis to pelvic LN. Future analysis of larger cohorts will help establish the biologic significance of prostate cancer metastasis to periprostatic LN.
\end{abstract}

\section{Keywords}

prostate cancer, prostatectomy, periprostatic lymph node, metastasis, biochemical recurrence

\section{Introduction}

Metastasis to pelvic lymph node (LN) in prostate cancer $(\mathrm{PCa})$ is well known to be associated with poor prognosis. ${ }^{1}$

Only few studies have addressed the clinical implications of PCa metastasis to periprostatic LN. The reported incidence of identifying such nodes during pathologic examination of radical prostatectomy specimens ranges from $0.8 \%$ to $4.4 \% .^{2-4}$ One prior study suggested that the presence of periprostatic LN metastases may have adverse prognosis compared with PCa without any LN metastasis. ${ }^{3}$ However, the prognostic significance of positive periprostatic LN compared with pelvic LN metastasis is not well defined. Direct comparison between metastases to pelvic and periprostatic LN has been limited by the relative infrequency of finding periprostatic LN during pathologic examination.

The purpose of our study is to document prognostic significance of metastasis to periprostatic LN, using biochemical recurrence (BCR)-free survival as an end point,

\footnotetext{
IJohns Hopkins Medical Institutions, Baltimore, MD, USA

${ }^{2}$ Hacettepe University School of Medicine,Ankara, Turkey

Corresponding Author:

George J. Netto, Johns Hopkins Hospital, 40 I N. Broadway/2242

Weinberg, Baltimore, MD 2I23I, USA.

Email:gnettol@jhmi.edu
} 
in comparison with pelvic LN metastasis, in a cohort study in which both groups are matched for age, Gleason score, and pathological stage.

\section{Material and Methods}

The study was approved by the Johns Hopkins Hospital Institutional Review Board.

\section{Population and Study Design}

Our surgical pathology database was searched for all radical prostatectomy patients with reported identification of any periprostatic LNs between 1984 and 2011. We identified 61 cases of a total of 20685 radical prostatectomy specimens, 19 of which showed metastatic carcinoma in at least one periprostatic node. Two of these cases also showed positive pelvic LN and were therefore excluded. Fifteen patients had follow-up information available regarding BCR status (group 1). Patients in group 1 were matched 1:2 to patients with positive pelvic LN (group 2) with exact matching on Gleason score, pathological stage, age-group, and race. Three patients in group 1 could only be matched to one patient in group 2 each. This resulted in an analysis cohort of 15 patients with positive periprostatic LN and 27 patients with positive pelvic LN.

All prostatectomy specimens were routinely processed according to our departmental protocol. Briefly, resected specimens were fixed in formalin, sliced at 3-mm intervals, and embedded entirely. All available hematoxylin and eosin slides from the cases were reevaluated. Histopathological characteristics, including Gleason score, margin status, pathological stage, size of periprostatic and pelvic lymph nodes, and their metastatic foci, were assessed by a urologic pathologist on the study. LNs were defined according to the presence of a capsule and other structural elements such as subcapsular sinus and follicles to differentiate from tumor-associated lymphoid infiltrates in perpendicularly transected extraprostatic tumor extension. Clinical information, including age, race, preoperative serum prostate-specific antigen (PSA) and BCR status were also collected.

\section{Statistical Analysis}

Comparisons of demographic and clinical characteristics between the 2 groups were performed using the $\chi^{2}$ test, Fisher's exact test for categorical variables, and the Wilcoxon rank sum test for continuous variables. Survival times were defined from the date of surgery and were represented by integer values. Biochemical recurrence was defined by the occurrence of post-radical prostatectomy serum PSA $\geq 0.2 \mathrm{ng} / \mathrm{mL}$, or the administration of salvage radiation with a detectable PSA (1 patient). BCR-free survival was estimated using the KaplanMeier method and survival curves were compared using the log-rank test. The stratified Cox proportional hazards model was used, with strata defined by the matched triplets. The independent variable was lymph node status (periprostatic vs pelvic LN). A robust sandwich estimator of the covariance matrix was used. ${ }^{5}$ Sample size needed for the Cox proportional hazards model was calculated using the method of George and Desu. ${ }^{6}$ A $P$ value $<.05$ indicated statistical significance. Analyses were performed with SAS version 9.3 (SAS Institute, Cary, $\mathrm{NC})$.

\section{Results}

The clinicopathological features of patients in both groups are summarized in Table 1. Preoperative serum PSA levels did not differ between the 2 groups. A single periprostatic node harboring metastatic $\mathrm{PCa}$ was identified in each of 14 cases. In the remaining case, 2 periprostatic nodes were identified with 1 of the 2 harboring metastasis. The laterality distribution of periprostatic LN was as follows: left $(\mathrm{n}=3)$, right $(\mathrm{n}=7)$, and unspecified $(\mathrm{n}=5)$. Location in relation to the prostate was available for 10 cases; periprostatic LNs were identified in posterior base in 6 cases ( 1 left and 5 right), mid posterolateral in 3 ( 2 left and 1 right), and in anterior apex in 1 case.

In group 1, the mean size of periprostatic LN was 1.24 $\mathrm{mm}$ with greatest dimension range of 0.9 to $3.2 \mathrm{~mm}$. Average greatest dimension of metastatic foci in periprostatic nodes was $0.7 \mathrm{~mm}(0.03-2.6 \mathrm{~mm})$. None of these were single cell or isolated tumor cell metastasis. In group 2 , pelvic $\mathrm{LN}$ and their metastatic foci measured, on average, $6.4 \mathrm{~mm}(2.5-12 \mathrm{~mm})$ and $2.6 \mathrm{~mm}(0.5-10 \mathrm{~mm})$ in greatest dimension, respectively. Patients with positive pelvic LN had significantly larger lymph nodes and larger metastases than those with positive periprostatic LN $(P=$ .0002 and $P=.007$, respectively).

Surgical margin status was significantly different between the 2 groups $(P=.024)$. Fifty-three percent of patients in group 1 showed positive margins. Of these 8 cases, the posterolateral margin was involved in 3 cases and the apical margin in other 3 ; one case showed involvement of both surgical margins. Only $19 \%$ of patients in group 2 showed positive margins.

The median follow-up was 1.0 year (range 1-13 years) for patients with positive periprostatic $\mathrm{LN}$ and 2.0 years (range 1-9 years) for patients with positive pelvic LN. The Kaplan-Meier BCR-free survival estimates in both groups are shown in Figure 1. At 5 years after surgery, $26 \%$ of patients in group 2 remained free of BCR whereas $69 \%$ of those in group 1 were free of BCR at that time. The latter difference did not reach statistical significance $(P=.072)$. 
Table I. Characteristics of Patients With Positive Periprostatic Lymph Nodes (Group I) and Matched Patients With Positive Pelvic Lymph Nodes (Group 2).

\begin{tabular}{|c|c|c|c|}
\hline Characteristic & Group I; n (\%) & Group 2; n (\%) & $P$ \\
\hline Age in years; median (range) & $6 I(5 \mid-69)$ & $60(50-69)$ & .598 \\
\hline \multicolumn{4}{|l|}{ Age-group in years } \\
\hline $50-59$ & $6(40)$ & II (4I) & \multirow[t]{2}{*}{.963} \\
\hline $60-69$ & $9(60)$ & $16(59)$ & \\
\hline \multicolumn{4}{|l|}{ Race } \\
\hline White & 13 (87) & $24(89)$ & \multirow[t]{2}{*}{.765} \\
\hline Non-white & $2(13)$ & $3(I I)$ & \\
\hline Preoperative serum PSA (ng/mL); median (range) & $6.8(3.4-24.3)$ & $6.3(2.9-62.2)$ & .731 \\
\hline \multicolumn{4}{|l|}{ Radical prostatectomy Gleason score } \\
\hline $3+4$ & $3(20)$ & $6(22)$ & \multirow[t]{4}{*}{.997} \\
\hline $4+3$ & $7(47)$ & $12(44)$ & \\
\hline 8 & $3(20)$ & $5(18)$ & \\
\hline 9 & $2(13)$ & $4(15)$ & \\
\hline \multicolumn{4}{|l|}{ Radical prostatectomy stage } \\
\hline $\mathrm{PT} 2 / 2 \mathrm{X}$ & $2(13)$ & $2(7)$ & \multirow[t]{3}{*}{.882} \\
\hline рT3a & $10(67)$ & $19(70)$ & \\
\hline PT3b & $3(20)$ & $6(22)$ & \\
\hline \multicolumn{4}{|l|}{ Positive surgical margins } \\
\hline Yes & $8(53)$ & $5(19)$ & \multirow[t]{2}{*}{.024} \\
\hline No & $7(47)$ & $22(8 \mathrm{I})$ & \\
\hline Size of lymph node in mm; median (range) & $0.8(0.1-3.2)$ & $5.5(2.5-12.0)$ & .0002 \\
\hline Size of metastasis in mm; median (range) & $0.1(0.03-2.6)$ & $\mathrm{I} .0(0.5-10.0)$ & .007 \\
\hline \multicolumn{4}{|l|}{ Year of surgery } \\
\hline $1992-1999$ & $4(27)$ & $5(20)$ & \multirow[t]{3}{*}{.782} \\
\hline $2000-2005$ & $5(33)$ & II (44) & \\
\hline $2006-2011$ & $6(40)$ & $9(36)$ & \\
\hline \multicolumn{4}{|l|}{ Biochemical recurrence } \\
\hline Yes & $4(27)$ & $16(59)$ & \multirow[t]{2}{*}{$.072^{\mathrm{a}}$} \\
\hline No & II (73) & II (4I) & \\
\hline \multicolumn{4}{|l|}{ Adjuvant therapy } \\
\hline Yes (ADT + XRT) & I (9) & I (3) & \multirow[t]{2}{*}{.454} \\
\hline No & $8(91)$ & $25(97)$ & \\
\hline
\end{tabular}

Abbreviations: PSA, prostate-specific antigen;ADT, adjuvant hormonal therapy; XRT, adjuvant radiation therapy. ${ }^{a} P$ value based on log-rank test of Kaplan-Meier biochemical recurrence-free survival curves.

All recurrences in group 1 occurred within 1 year. There was no significant difference in adjuvant therapy between the 2 groups $(P=.454)$.

Hazard ratios were estimated using stratified Cox proportional hazards models (Table 2). Model 1 estimated the univariate hazard ratio for both groups. Patients in group 1 had a $38 \%$ lower risk of BCR than patients in group 2; however, the finding did not reach statistical significance $(P=.245)$. Model 2 adjusted for effect of preoperative serum PSA levels. Patients in group 1 showed a 33\% lower risk of BCR than those in group $2(P=.349)$. Model 3 adjusted for effect of surgical margin status. Although the magnitude of reduced risk in patients with positive periprostatic LN was larger (41\% reduction in risk), the findings were not statistically significant $(P=.216)$. Models 4 and 5 adjusted for size of lymph nodes and size of metastasis, respectively. Once more, patients with positive periprostatic LN had lower risk of BCR $(0.20$ and 0.44 , respectively). The findings again lacked statistical significance $(P=.677$ and $P=.249$, respectively). Adjustment for residual differences in age or year of surgery led to similar results as in Model 1 (data not shown).

\section{Discussion}

The literature on periprostatic LN metastasis is limited, with the few published studies reporting a very low incidence. ${ }^{3,4}$ Periprostatic LNs are relatively small in size, with an average diameter of $1.2 \mathrm{~mm}$ found in the present study and therefore not readily identifiable during gross 


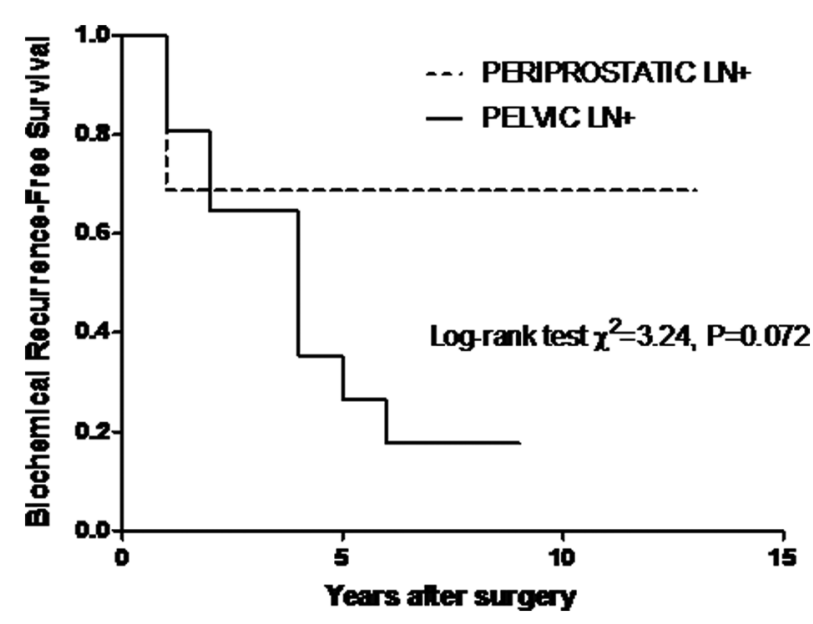

Figure I. Kaplan-Meier biochemical recurrence (BCR)-free survival estimates in patients with metastasis to periprostatic (group I) and pelvic (group 2) lymph nodes. All recurrences in group I occurred within I year.At 5 years after surgery, 69\% of patients in group I were free of BCR whereas $26 \%$ of those in group 2 remained free of BCR at that time.

examination. Extent of sampling during pathological examination of radical prostatectomy could also be a factor in the rate of their identification. As a result, the identification of metastasis to these nodes will be affected by the yield of periprostatic lymph node harvest, which in turn is related to the amount of periprostatic soft tissue removed during surgery.

It has been well documented that the predominant lymphatic drainage of the prostate is to obturator fossa and adjacent external and internal iliac lymph nodes that are most commonly examined after pelvic lymph node dissection in association with radical prostatectomy.

Separate, albeit rare, routes of minor lymphatic drainage to periprostatic LN could explain the phenomenon of metastasis to these nodes. Using a single photon emission computed tomography system after transrectal intraprostatic injection of radioactive tracers, periprostatic LN were identified as sentinel lymph nodes in only $0.3 \%$ of patients with high-grade $\mathrm{PCa}{ }^{7}$

Similar to previous reports, most of the positive periprostatic LN in our study were located near the posterior base. $^{2-4}$ This is also in line with previous finding of significantly high lymphatic density in the mid-base region, around the ejaculatory ducts. ${ }^{2,4}$ We also found 1 case with positive periprostatic LN in the anterior apex. A recent study showed that dissection and careful microscopic examination of anterior fibroadipose tissue revealed small lymph nodes in approximately $17 \%$ of patients with an overall metastasis rate of $2.5 \%{ }^{8}$ We found periprostatic nodes to be significantly smaller in size in our cases in comparison with pelvic nodes in controls. The same was true for size of metastases in both groups.
In the study by Kothari et al, ${ }^{2}$ all 3 patients with isolated PCa metastasis to periprostatic LN recurred between 4 and 7 months postprostatectomy. Based on such findings, Srigley ${ }^{9}$ suggested that patients with metastatic $\mathrm{PCa}$ to periprostatic $\mathrm{LN}$ should be staged as $\mathrm{pN} 1$. In a more recent study, positive periprostatic LN have been linked to larger tumor volume, higher stage, higher Gleason score, higher recurrence rate, and shorter time to recurrence compared with patients with negative periprostatic nodes. $^{3}$

Matching for relevant clinicopathological characteristics is a strength in our study that allowed us to minimize the effect of potential confounding parameters on BCR, including age, Gleason score, stage, and year of surgery. Our finding of significantly higher incidence of positive margin in patients with positive periprostatic LN compared with patients with positive pelvic LN is intriguing. However, it remains unclear whether the higher rate of positive margins in our cases is reflective of underlying biological behavior given that margin status did not affect BCR in our cases.

Although our analysis suggested that metastasis to periprostatic LN may be associated with a $30 \%$ to $40 \%$ BCR risk reduction, the association lacked statistical significance and should be interpreted with caution given the small sample size and the fact that our study remains underpowered. The results, however, warrant further investigation with larger patient cohort and longer followup. This is especially true considering the variance of our findings with prior studies and their possible implication on pathological handling of specimens, substaging of tumor, and treatment strategies. 


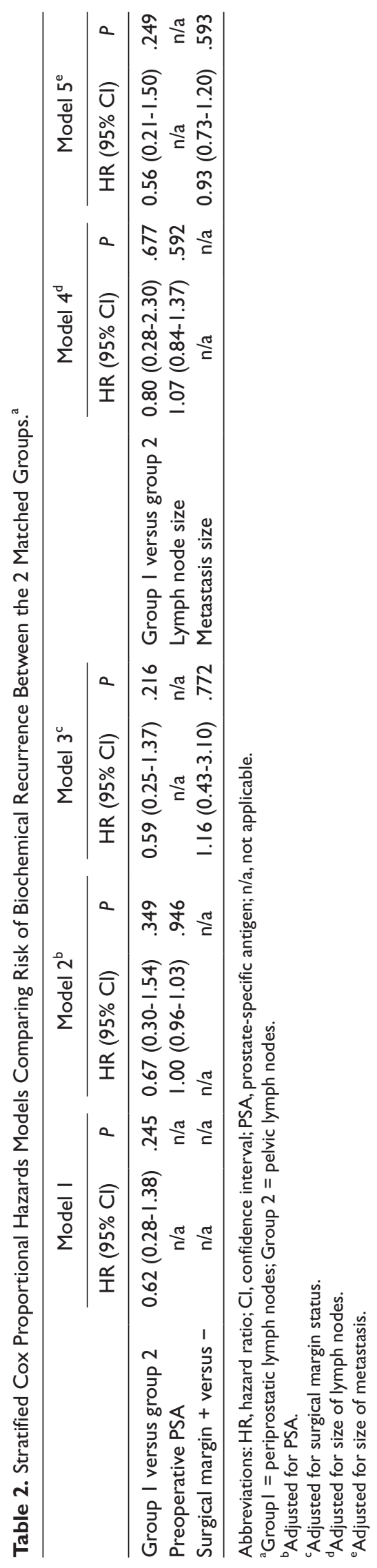




\section{Authors' Note}

Nilda Gonzalez-Roibon and Jeong S. Han contributed equally to this study.

\section{Declaration of Conflicting Interests}

The author(s) declared no potential conflicts of interest with respect to the research, authorship, and/or publication of this article.

\section{Funding}

The author(s) disclosed receipt of the following financial support for the research, authorship, and/or publication of this article: This study was partially supported by the Johns Hopkins Medicine Patana Fund for Research.

\section{References}

1. Adams J, Cheng L. Lymph node-positive prostate cancer: current issues, emerging technology and impact on clinical outcome. Expert Rev Anticancer Ther. 2011;11:1457-1469.

2. Kothari PS, Scardino PT, Ohori M, Kattan MW, Wheeler TM. Incidence, location, and significance of periprostatic and periseminal vesicle lymph nodes in prostate cancer. $\mathrm{Am}$ J Surg Pathol. 2001;25:1429-1432.
3. Deng FM, Mendrinos SE, Das K, Melamed J. Periprostatic lymph node metastasis in prostate cancer and its clinical significance. Histopathology. 2012;60:10041008.

4. Subik MK, Yao JL, di Sant'Agnese PA, Miyamoto H. The role of periprostatic and periseminal vesicle lymph node metastasis in the staging and prognosis of prostate cancer. Histopathology. 2012;60:1009-1010.

5. Lin DY, Wei LJ. The robust inference for the proportional hazards model. J Am Stat Assoc. 1989;84:1074-1078.

6. George SL, Desu MM. Planning the size and duration of a clinical trial studying the time to some critical event. $J$ Chronic Dis. 1974;27:15-24.

7. Ganswindt U, Schilling D, Müller AC, Bares R, Bartenstein P, Belka C. Distribution of prostate sentinel nodes: a SPECT-derived anatomic atlas. Int $J$ Radiat Oncol Biol Phys. 2011;79:1364-1372.

8. Yuh B, Wu H, Ruel N, Wilson T. Analysis of regional lymph nodes in periprostatic fat following robot-assisted radical prostatectomy. BJU Int. 2012;109:603-607.

9. Srigley JR. Key issues in handling and reporting radical prostatectomy specimens. Arch Pathol Lab Med. 2006;130: 303-317. 